\title{
Analysis of the Influence of Track Lateral Irregularity on the Stability of Seamless Turnout
}

\author{
Yuan LI, Feng HAN ${ }^{1}$, Miaomiao QI, Qiang JIA, and Tao LIANG \\ College of Civil Engineering, Lanzhou Jiaotong University, Lanzhou 730070, China
}

\begin{abstract}
With the rapid development of the Sino-Russian railway, in order to study the influence of track lateral irregularities on the deformation of seamless turnouts in alpine regions, a nonlinear finite element model of the spatial track frame was established to study the influence of track lateral irregularities on the stability of seamless turnouts. By setting parameters, the effects of temperature changes, lateral irregularities in the switch part, and lateral irregularities in the guide curve on the stability of the seamless turnout are studied. The results show that the greater the temperature change, the greater the lateral displacement. By comparing various working conditions, it can be concluded that when the curved rail of the guide curve part has lateral deviation, the seamless turnout will have a large lateral deformation, and the lateral displacement of the straight basic rail has a more obvious increase.
\end{abstract}

Keywords. Seamless turnout, nonlinear finite element analysis, stability, lateral irregularities, lateral displacement

\section{Introduction}

As one of the key technologies of seamless turnout, there are extremely complex loadbearing, power-transmission and deformation relations among the rails, which directly affect the strength, stability and traffic safety of the seamless turnout [1]. The forces of seamless turnouts in alpine and high-altitude areas are more complicated. The SinoRussian railway is located in the alpine region, and the seamless turnout will produce a certain degree of horizontal irregularity under the action of temperature force. The seamless turnout is more obviously affected by the temperature force. With the change of temperature, the rail will stretch, and the fastener will resist the displacement change of the rail. If the force exceeds the limit, the track of expansion will occur, affecting the stability of the line. Many experts at home and abroad have studied the influence of different factors on the stability of Seamless turnout. Xu Shiru and others [2] analyzed the temperature force distribution and deformation of jointless turnout; Xu Qingyuan and others [3-5] analyzed the influence of track parameters on jointless turnout combination effect, studied the longitudinal force and displacement of jointless turnout rail with finite element method, and obtained the calculation model of additional force of jointless track on bridge; Gao Liang and others [6] analyzed the spatial mechanical characteristics of seamless turnout on bridge; Tang Jinfeng and others [7,8] analyzed

\footnotetext{
${ }^{1}$ Corresponding Author, Feng Han, Pucheng, Shanxi, PhD, professor. E-mail : 153025377@qq.com.
} 
the effect of temperature gradient; Japan Yanagawa Hideaki [9] researched under different initial bending curve radius and the stability of the seamless line problem, Feng Qingsong and others [10] was studied under the action of temperature force seamless line drum up instability problem, when the initial bending is $1 \mathrm{~cm}$, the former temperature $50{ }^{\circ} \mathrm{C}$ lateral displacement is roughly $1 \mathrm{~mm}$, the latter temperature $50{ }^{\circ} \mathrm{C}$ lateral displacement is roughly $2 \mathrm{~mm}$, more than the displacement limit line will likely damage; Yan Le and others [11] analyzed the influence of slope on the stress and deformation of jointless turnout on long ramp bridge; Zhou Haiyu and others [12,13] studied the influence of track irregularity on the stability of CWR; but they did not analyze the stability of jointless turnout in turnout area of subgrade section under the action of temperature force. Especially in permafrost regions, the subgrade section has such problems as large daily temperature difference, changeable climate, and many large and long ramps [14]. The horizontal irregularities of the welded turnout in the alpine region are very unfavorable to the stability and maintenance of the welded rail in the turnout area. Therefore, studying the law of the lateral displacement of the long rail of the turnout under the changes of various factors is of guiding significance in solving the stability problem of the seamless turnout in the high-cold permafrost area.

\section{Establishment of 3D Space Model of Seamless Turnout}

\subsection{Mechanical Model}

Based on the assumption of small deformation, the nonlinear finite element is studied in this paper, and the track frame is regarded as a slender pressure bar laid in uniform medium, and the three-dimensional model of seamless turnout with No.12 movable center rail is established. The track frame model with $60 \mathrm{~kg} / \mathrm{m}$ rails, elastic bar type II fastener and concrete type II sleeper. The sleeper 1840 is laid per $\mathrm{km}$, and the width of track sleeper shoulder is $40 \mathrm{~mm}$. The radius of guide curve is $350.7175 \mathrm{~m}$, the transverse resistance stiffness of ballast sleeper is $1500 \mathrm{~N} / \mathrm{mm}$, the longitudinal resistance stiffness of ballast sleeper is $2500 \mathrm{~N} / \mathrm{mm}$, and the vertical stiffness is $3200 \mathrm{~N} / \mathrm{mm}$. A seamless turnout model is established, which takes the rail, sleeper, fastener and ballast resistance as an integral whole. The rail and sleeper are simulated by spatial beam elements with different material properties, and are divided by the support points of the sleeper. The fastener simulates the connection with rail and sleeper with the spring constraint of longitudinal, transverse and torsion three degrees of freedom. The lateral, longitudinal and vertical action of ballast sleeper resistance is simulated as a spring element, and its action is also used as the medium connecting sleepers and roadbed, so the interaction effect can be fully considered in force analysis.

A pair of limiter with a limit value of $7 \mathrm{~mm}$ was set at the heel end of the cusp. After the letter block of the limiter was closely attached, it was selected as $14 \mathrm{kN} /{ }^{\circ} \mathrm{C}$ in reference to literature15, and was added to the model in the form of a pair of nodal forces. At the heel end of the core rail, the increase in the resistance of the spacer iron is taken as $2.9 \mathrm{kN} /{ }^{\circ} \mathrm{C}$ with reference to the literature 16 , which is added to the adjacent nodes of the wing rail in the form of nodal force to simulate the force transmission between the core rail and the wing rail. 


\subsection{The Solution Method of Nonlinear Finite Element}

Under the conditions assumed by Kirchhoff, the strain of the beam element of the space beam is composed of linear strain and nonlinear strain [15]. When solving nonlinear problems, the relationship between load and nonlinear displacement can generally be regarded as a series of linear responses.

Generally, Lagrangian column method is used to solve nonlinear finite element problems [7]. A complete iterative step is as follows:

- Measure the irregularity of the line and assign the value according to the measured initial displacement;

- According to linear analysis, the initial value of node displacement is obtained $\{\delta\}_{1}$;

- Construct element tangent stiffness matrix $\left[K_{T}\right]$ in local coordinate system, and calculate element nodal force $[F]$;

- Convert $\left[K_{T}\right]$ and $[F]^{\mathrm{e}}$ to a global coordinate system;

- Repeat steps 3 and 4 for all the elements to generate the structural tangent stiffness matrix $\left[K_{p}\right]_{1}$ and nodal force vector $[F]_{1}$;

- Calculate the unbalanced forces;

$$
\left\{P_{1}\right\}=\{F\}_{1}-\sum\{F\}^{e}
$$

- The structural stiffness matrix equation 2 is solved to obtain the displacement increment $\triangle\{\delta\}_{1}$ of the node;

$\left[K_{P}\right]_{1} \bullet \Delta\{\delta\}_{1}=\{P\}_{1}$

- Add $\triangle\{\delta\}_{1}$ to the structure displacement vector $\{\delta\}_{1}$, namely

$$
\{\delta\}_{2}=\{\delta\}_{1}+\Delta\{\delta\}_{1}
$$

- Judge the convergence condition, if not satisfied, go back to step 3.

$$
\frac{\sqrt{\left[\Delta\{\delta\}_{m}\right]^{T} \cdot\left[\Delta\{\delta\}_{m}\right]}}{\sqrt{\left[\{\delta\}_{m}\right]^{T} \cdot\left[\{\delta\}_{m}\right]}} \leq e
$$

\section{Analysis of Influencing Factors of Seamless Turnout Stability}

\subsection{Displacement Variation under Different Temperature Rise Conditions}

Based on the calculation and analysis of the established track frame model, the overall structure of the seamless turnout is heated by $10^{\circ} \mathrm{C}, 20^{\circ} \mathrm{C}, 30^{\circ} \mathrm{C}, 40^{\circ} \mathrm{C}$, and $50^{\circ} \mathrm{C}$, and the law of its lateral and longitudinal displacement changes with temperature changes is solved. 


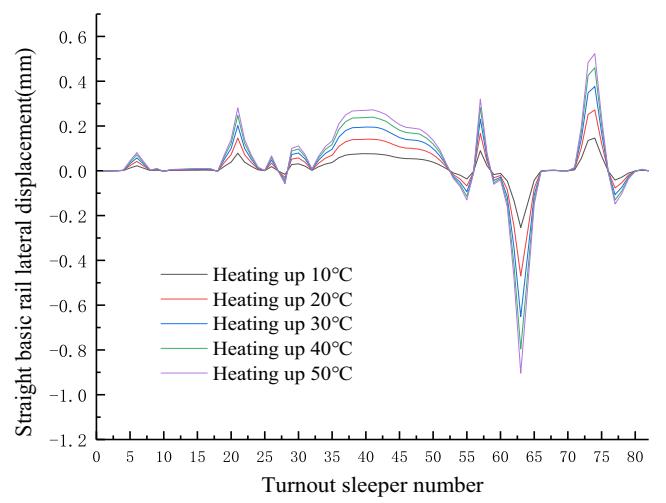

Figure 1. Trend of lateral displacement of straight basic rail

The change trend of the trajectories of the welded turnout is similar when the temperature rises, and the direction of the lateral displacement is that the deviation to the outside of the straight basic rail is positive, and the deviation to the inside is negative. The stress characteristics of the straight basic rail are relatively obvious. Under different temperature rising conditions, its transverse displacement variation trend is shown in Figure 1. The position of each unfavorable turnout tie is shown in Figure 2. The maximum lateral displacement changes from the bifurcation center to the end of the wing rail. The higher the temperature is, the greater the lateral displacement of the rail is.

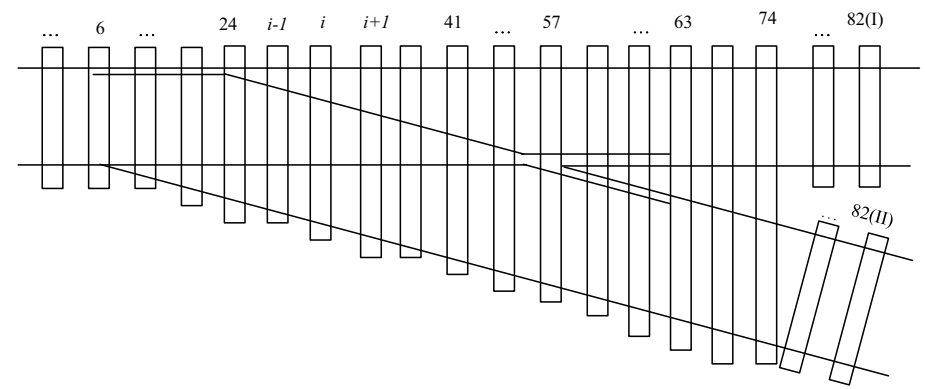

Figure 2. Schematic diagram of bifurcated pillows at unfavorable positions

\subsection{Transverse Irregularities of the Orbit}

The causes of track lateral unevenness include the track centerline positioning deviation caused by the track laying construction and the whole track operation, the lateral residual deformation accumulation of the rail row, the uneven wear of the side of the rail head, the failure of the fasteners, and the inconsistent lateral elasticity of the track. The track direction is uneven, and the gauge deviation is caused by poor fasteners, rail head side wear, sleeper shoulder failure and other reasons. This deviation is equivalent to imposing a relatively large initial irregularity on the track structure. When the internal temperature and pressure of the rail is large, it is very likely to produce an expanded track under the influence of the initial irregularity, which will threaten the safety of train operation. 
Based on the calculation model established in this paper, forced lateral displacement is generated at each unfavorable position point. Because of the continuity of the long rail, when one point is displaced, it will inevitably drive the displacement of adjacent nodes. In the calculation, the lateral displacement of adjacent nodes must be calculated. Displacement is applied to the model at the same time, so as to simulate the uneven working condition in the line. Given the heating condition of $50^{\circ} \mathrm{C}$, the deformation of the turnout only under the action of the temperature force is compared as the normal working condition, and the change law of the lateral displacement of the seamless turnout under the condition of the track lateral irregularity is analyzed.

\subsubsection{Horizontal Irregularity of Straight Basic Rail}

Take the transverse irregularity of turnout at No. 21 turnout as an unfavorable condition.

- The basic rail is externally shifted by $1 \mathrm{~mm}$ at the 21 fork pillow

- Straight basic rail and curved basic rail are simultaneously offset $1 \mathrm{~mm}$ outwards at 21 turnout sleepers

Table 1. Trend of Transverse Displacement of the Straight Basic Rail at the Switch

\begin{tabular}{ccccc}
\hline Working condition of type & \multicolumn{3}{c}{ Peak point position of straight base rail } \\
\cline { 2 - 4 } & Node 6 & Node 8 & Node 19 & Node 21 \\
\hline $\begin{array}{c}\text { Normal condition } \\
\text { Straight basic rail deviates 1 mm } \\
\text { outside ( condition 1) }\end{array}$ & 0.083 & -0.007 & -0.019 & 0.281 \\
$\begin{array}{c}\text { Straight basic track and curved basic } \\
\text { track 1 mm outside (condition 2) }\end{array}$ & 0.090 & -0.008 & -0.021 & 0.315 \\
\hline
\end{tabular}

It can be seen from Table 1 that under the first condition, the lateral displacement of the rail has a tendency to increase, and the lateral displacement generated at the No. 21 turnout tie is $0.034 \mathrm{~mm}$ larger than the normal condition, which is an increase of $12.01 \%$. Under the second condition, the lateral displacement of the straight basic rail increases by $0.051 \mathrm{~mm}$, an increase of $18.15 \%$. It is concluded that the transverse deformation of the structure is greater under the action of temperature force when the lateral unevenness of the two rails is at the same bifurcation pillow than that of the single rail. According to the railway line repair rules, the distance between the sharp rail and the basic rail shall not exceed $1 \mathrm{~mm}$. The sharp rail is relatively weak, and due to the existence of track irregularities, the sharp rail is likely to produce side bending, thus making the gauge not in accordance with the provisions. Therefore, it is necessary to regularly detect the point part, especially the geometric shape of the line at the end of the pointed track and the normal use of all parts to prevent the occurrence of adverse working conditions.

\subsubsection{Lateral Irregularity of Monorail Rail}

Under the action of temperature force, the transverse displacement peak of straight basic rail, curved guide rail, straight guide rail and curved basic rail in the guide curve of seamless turnout was obtained at the pillow of No. 41 turnout. 41 bifurcation pillow is chosen as the most unfavorable horizontal kinds of track irregularities, and calculation conditions, set the temperature of $50^{\circ} \mathrm{C}$, respectively, the four rail lines produce $1 \mathrm{~mm}, 2 \mathrm{~mm}, 3 \mathrm{~mm}$ outside migration, using forced displacement method, for 
the disadvantage point trajectory track horizontal rough chronological influence of seamless rail lateral deformation.

Straight guide rail and a curved lateral displacement less change, so a straight rail and curved rail, for example, can be seen in Figures 3 and 4, when the straight outer rail $1 \mathrm{~mm}$ shift fork 41 at the nut, at a temperature of force, Straight basic rail, curved guide rail, straight guide rail, curved basic rail (hereinafter referred to as four-track line in this order) peaks are $0.361 \mathrm{~mm}, 0.363 \mathrm{~mm}, 0.360 \mathrm{~mm}, 0.363 \mathrm{~mm}$, respectively, which are $0.088 \mathrm{~mm}$ larger than normal operating conditions, $0.088 \mathrm{~mm}, 0.087 \mathrm{~mm}, 0.089 \mathrm{~mm}$, respectively, an increase of $32.2 \%, 32 \%, 31.7 \%, 32.48 \%$. When the lateral offset of $2 \mathrm{~mm}$, four lateral displacement trajectory peaks were increased by $88.28 \%, 76 \%$, $76.56 \%, 75.91 \%$. When the lateral offset of $3 \mathrm{~mm}$, four lateral displacement trajectory peaks were increased by $141 \%, 137.45 \%, 136.63 \%, 136.13 \%$.

When the curved basic rail is shifted to the outside by $1 \mathrm{~mm}$, the lateral displacement peak of the four-rail line at the 41 bifurcated pillow increases by $0.079 \mathrm{~mm}, 0.078 \mathrm{~mm}, 0.080 \mathrm{~mm}$ and $0.080 \mathrm{~mm}$, respectively, compared with the normal condition, with increases of $28.93 \%, 28.36 \%, 29.3 \%$ and $29.1 \%$, respectively. When the external deviation is $2 \mathrm{~mm}$, the peak increase of lateral displacement of four-track is $75.46 \%, 75.55 \%, 75.82 \%$ and $75.78 \%$, respectively. When the external offset is $3 \mathrm{~mm}$, the peak lateral displacement of the four-track increases by $137 \%, 133.09 \%, 133.7 \%$ and $133.2 \%$, respectively.

From the calculation results: When there is track lateral irregularity in the guide curve, the lateral deformation of the rail under the same heating condition is more obvious than the normal working condition, and the lateral displacement peak value is significantly increased. The greater the value of the rail's lateral irregularity, the greater the lateral deformation of the rail, and with the increase of the irregularity, the greater the increase in lateral displacement. When the trajectory irregularity increases to a certain degree, the lateral displacement of the outer straight basic rail has a significant increase. Under the same temperature change conditions, its displacement value increases most obviously. Under the most unfavorable conditions, the increase ratio can be Up to $137 \%$.
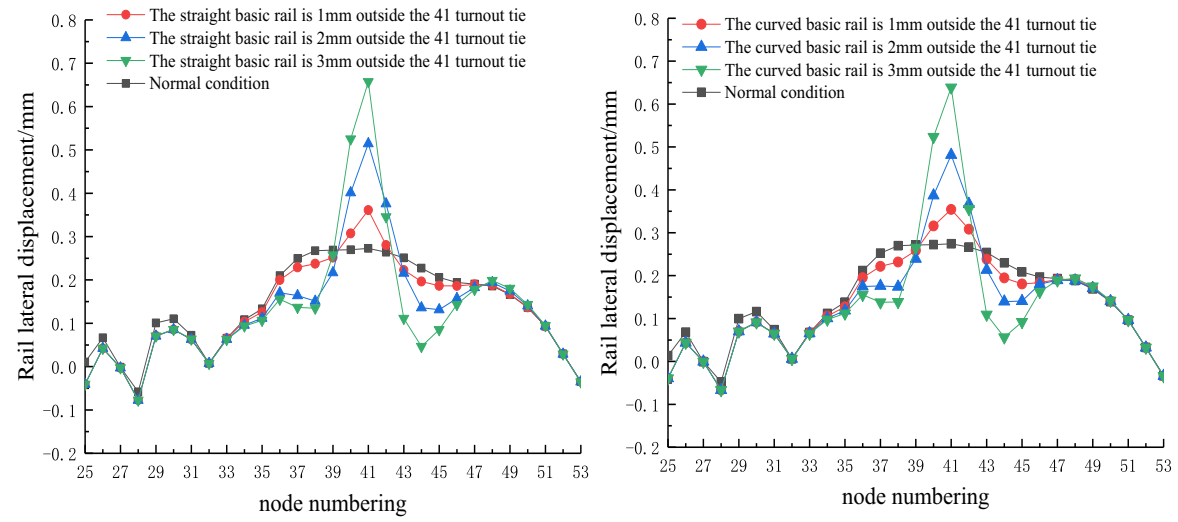

Figure 3. Trend of lateral displacement of straight basic rail (Left)

Figure 4. Trend of lateral displacement of curved basic track (Right)

The comparison shows that when the outer basic rail has irregularities, the lateral displacement increment of the remaining trajectories is inversely proportional to the 
distance between the outer rails, that is, the closer to the rail with irregularity, the greater the degree of influence, if the irregularity When the degree appears on the inner guide rail, the influence on the adjacent two rails is similar.

\subsubsection{Transverse Irregularity of Straight Strand Rail}

The guide curve is an important part of the seamless turnout. The curved rail is composed of the curved rail and the curved basic rail. Its function is to guide the train to go through laterally, while the straight rail is composed of the straight basic rail and the straight rail to guide the vehicle to go through directionally.

- The straight basic rail and the straight guide rail are offset $1 \mathrm{~mm}$ outwards at 41 turnout sleepers

- Straight strand straight basic rail and straight guide rail are offset $1 \mathrm{~mm}$ inward on 41 turnout sleepers
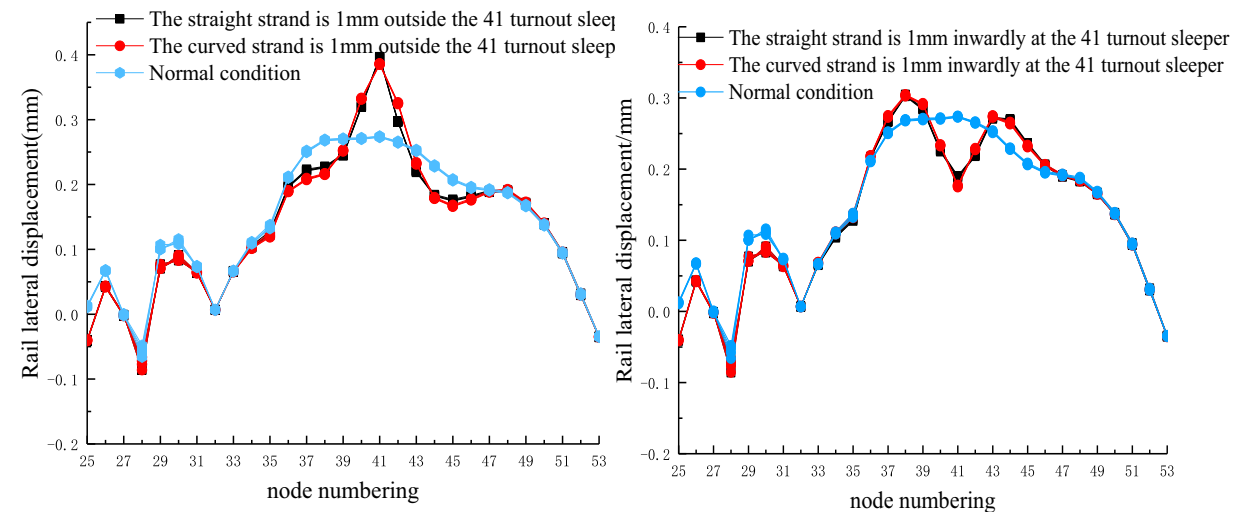

Figure 5. Straight basic rail lateral displacement (Left)

Figure 6. Straight basic rail lateral displacement (Right)

It can be seen from figure. 5 and 6 that when the straight and curved strands of the guide curve are offset by $1 \mathrm{~mm}$ outside and inside the No. 41 turnout sleeper, under the action of temperature, the degree of deformation of each trajectory is increased compared with the normal working condition. Larger, the lateral displacement peak also increases.

Table 2. Peak lateral displacement of each four-track line at 41 turnout tie (mm)

\begin{tabular}{ccccc}
\hline Condition type & Straight base rail & Curved rail & Straight rail & Basic track \\
\hline $\begin{array}{c}\text { Normal condition } \\
\text { Straight strand outer } \\
\text { deviation 1 mm }\end{array}$ & 0.273 & 0.275 & 0.273 & 0.274 \\
$\begin{array}{c}\text { Outward deviation of } \\
\text { curved strands 1 mm } \\
\text { Straight strand inner } \\
\quad \begin{array}{c}\text { deviation 1 mm } \\
\text { 1mm inward deviation of } \\
\text { curved strands }\end{array}\end{array}$ & 0.395 & 0.397 & 0.396 & 0.397 \\
\hline
\end{tabular}

It can be seen from Table 2 that, when the straight strand has a lateral shift of $1 \mathrm{~mm}$ at the no. 41 bifurcated pillow, the straight basic rail, curved guide rail, straight guide rail and curved basic rail reach the peak transverse displacement at the no. 41 
bifurcated pillow, and the increase in the transverse displacement from the normal condition is $44.69 \%, 44.37 \%, 45.55 \%$ and $44.89 \%$, respectively. Therefore, in the guide curve part, when the straight and curved rail has lateral deviation of $1 \mathrm{~mm}$, the lateral deformation of the four-rail line under the heating condition is larger than the normal condition. The lateral unevenness of the straight strand rail will result in a large lateral displacement peak, and the peak value is greater than that of the curved strand rail. But for the adjacent nodes of the peak point, the increase of displacement generated by straight rail deviating is less than that of curved rail deviating.

When the straight strand has an inner shift of $1 \mathrm{~mm}$ at the no. 41 bifurcation pillow and the curved strand has an inner shift of $1 \mathrm{~mm}$ at the no. 41 bifurcation pillow, the variation trend of the lateral displacement of the four-rail line is concave parabola. The internal offset displacement value of curved rail decreases by $8.79 \%$ compared with that of straight rail, but the offset displacement value of curved rail increases by $6.74 \%$ and $10.57 \%$ respectively compared with that of straight rail at the position of no. 38 bifurcated pillow and no. 44 bifurcated pillow on both sides of the wave peak. In other words, when the rail of curved strand produces inner deviation, the change trend of transverse displacement into concave shape is more obvious, and curved strand provides the transverse nodal force for the turnout structure to produce transverse deformation. Therefore, when the rail has inner deviation, attention should be paid to the influence of the lateral displacement crest on the structure deformation caused by the concave variation trend. Domestic scholars Wang Ping and Liu Xueyi stated in Literature 16 that when the track heel is a spacer iron, the acting force is $550 \mathrm{kN}$, and the relative displacement between the guide rail and the basic rail is $5 \mathrm{~mm}$. The calculation in this paper is safer and conforms to the law.

\section{Conclusion}

Based on the space member system, this paper establishes the non-linear finite element model of No. 12 welded turnout. At the unfavorable positions of each part of the welded turnout, the influence factors of the track lateral irregularity are superimposed, and the transverse displacement of the welded turnout under various unfavorable conditions is analyzed. The development law of the jointless turnout, and then related analysis and demonstration of the influence of the seamless turnout stability.

- The calculation program verifies that under the action of temperature force, the seamless turnout will have a sudden change in lateral displacement at the tip of the switch rail, the heel end of the switch rail, the middle of the guide curve, the switch center to the end of the wing rail, and the separation point of straight side strands. According to the analysis of the peak point position, it can be known that the connection form and linear change of the important force transmission components that make up the stiffness of the track frame will directly affect the stiffness of the track frame, which will affect the stability of the seamless turnout. As it rises, the lateral displacement of the structure continues to increase.

- When seamless turnouts have track lateral irregularities at unfavorable locations, under the action of temperature forces, the lateral displacement of the rails is greater, and there is a tendency to develop along their respective offset directions. When there is lateral irregularity on the same turnout tie in 
the double track line, the lateral displacement of the turnout is greater when the lateral irregularity exists on the single track line, and with the increase of the irregularity, the greater the increase in the lateral displacement of the rail.

- In the guide curve part, the degree of influence between the trajectories is inversely proportional to the distance between the trajectories. When the irregularity of each trajectory increases to a certain extent, the lateral displacement produced by the outer straight basic rail will increase significantly. By making the straight strand rails and curved strand rails produce outer and inner deviations at unfavorable positions, the comparative analysis shows that when the curved strands produce outer deviations, it will have a greater impact on the deformation of the turnout. The degree of deformation shows that the structure will cause lateral deformation. The transverse nodal force of is mainly provided by the curved steel rail.

\section{Acknowledgement}

The author thanks the tutor and his National Natural Science Foundation of China (51568037) for their help in this article.

\section{References}

[1] Li CH. Rail. Chengdu: Southwest Jiaotong University Press, 2012, 2-3.

[2] $\mathrm{Xu} \mathrm{SR,} \mathrm{Tong} \mathrm{BH.} \mathrm{Temperature} \mathrm{Force} \mathrm{Distribution} \mathrm{and} \mathrm{Deformation} \mathrm{Analysis} \mathrm{of} \mathrm{Welded} \mathrm{Switches.}$ Journal of the China Railway Society, 1994(1):73-79.

[3] Xu QY, Zhou XL, Chen XF. Inf luence of track parameter on welded turnout combination system. Journal of Traffic and Transportation Engineering, 2003,3(2):7-10.

[4] Xu QY, Analysis of Longitudinal Force and Displacement of Seamless Turnout with Finite Element Methodology. China Railway Science, 2003,24(6):36-40.

[5] Xu QY, Zhou XL, Yang XY. Computation model of additional longitudinal forces of continuously welded rails on bridges. Journal of Traffic and Transportation Engineering, 2004(1):25-28.

[6] Gao L, Tao K, Qu C, Xin T. Study on the Spatial Mechanical Characteristics of Welded Turnout on the Bridges for Passenger Dedicated Lines. China Railway Science, 2009,30(1):29-35.

[7] Tang JF, Yin HT, Zeng ZP, Tang CG. Mechanical Characteristic of Slab Track on Switch area under Temperature Gradient. Journal of Railway Science and Engineering, 2011, 08(1):24-28.

[8] Zhao WH, Wang P, Cao Y, Additional Temperature Forces of Continuous Welded Rail on Large-span Steel Truss Cable-stayed Bridge//Southwest Jiaotong University. Proceedings of 3rd International Conference on Transportation Engineering. New York: American Society of Civil Engineers, 2011: 1767-1773

[9] Yanagawa H, Katatan H. Exploring Buckling Stability Analysis of Rossogrel. Railway Research Review, 2001, 57 (1): 18 - 21 Alternative Energy Source.

[10] Feng QS, Zong DM, Lei XY. Finite Element Model for Analyzing the Stability of Continuously Welded Rail Track. China Railway Science, 2005(01):8-15+25.

[11] Yan L, Xiong ZW, Wei XK, Wang P. Influence of Slope Gradient on Gapless Turnout upon Bridge with Long Heavy Grade. Railway Standard Design, 2013(9).

[12] Zhou HY. Analysis of the influence of track irregularity on the Stability of CWR. Lanzhou Jiaotong University, 2019.

[13] Zhou HY, Han F, Mu H. Influence of Track Lateral Irregularity on Continuous Welded Rail Deformation. Railway Engineering,2018,58(7): 116-120.

[14] Gao DF. Research on the CWR Stability of High Roadbed Section in Permafrost Regions. Lanzhou Jiaotong University,2015.

[15] Zhou HL, Zhou SX. The Derivation of Transforming Matrix in 3D Space Solid-Beam Element Method. China Railway Science, 2014,39(6): 147-151. 